\title{
Motivos de interés por la docencia e identidad profesional del futuro profesorado de Educación Física. Análisis en los másteres universitarios de Sevilla, Granada y Jaén (España)
}

\author{
Carolina CASTAÑEDA-VÁZQUEZ \\ Antonio J. PÉREZ-CORTÉS \\ Pedro A. VALDIVIA-MORAL \\ Félix ZURITA-ORTEGA
}

Datos de contacto:

Carolina Castañeda-Vázquez Departamento de Educación Física y Deporte, Universidad de Sevilla

carolinacv@us.es

\section{Antonio J. Pérez-Cortés}

Departamento de Didáctica de la Expresión Musical, Plástica y

Corporal, Universidad de Granada antperez@ugr.es

Pedro A. Valdivia-Moral Departamento de Didáctica de la Expresión Musical, Plástica y Corporal, Universidad de Granada

pvaldivia@ugr.es

Félix Zurita-Ortega

Departamento de Didáctica de la Expresión Musical, Plástica y Corporal, Universidad de Granada

felixzo@ugr.es

\section{RESUMEN}

La identidad profesional docente está determinada por los motivos de interés hacia la profesión, así como por los cambios en las actitudes y creencias de los futuros docentes. El presente estudio pretende conocer el interés por la profesión docente y los motivos que incitan al alumnado del Máster de Educación Secundaria (especialidad Educación Física) de las Universidades de Granada, Sevilla y Jaén (España), a decantarse hacia la profesión docente, así como sus ideas previas acerca de la formación inicial. Para ello se aplicó el Cuestionario sobre interés por la docencia y la formación inicial (CIDFI) (Pontes, Ariza y del Rey, 2010) a una muestra de 217 estudiantes ( 154 hombres y 63 mujeres, de entre 21 y 35 años). Los datos revelaron una tendencia vocacional predominante especialmente en las mujeres, considerando muy importantes los conocimientos de pedagogía, didáctica y programación docente, así como la adquisición de destrezas para motivar a los alumnos por el aprendizaje. Como propuesta de mejora, parece conveniente incluir dentro del máster actividades que permitiesen explicitar las ideas previas y motivaciones de los futuros docentes, así como estos datos deberían tenerse en cuenta en el diseño de la formación inicial, tanto general como específica, de los futuros docentes de Secundaria.

PALABRAS CLAVE: Formación Universitaria; Formación profesional docente; Educación Física; Educación Secundaria Obligatoria. 


\title{
Motives for Becoming a Teacher and Future Physical Education Teacher's Professional Identity. Analysis in the University Master's Degrees of Seville, Granada and Jaen (Spain)
}

\begin{abstract}
Teacher identity is determined both by the reasons that explain the interest in the profession and by the changes promoted in the attitudes and beliefs of the teacher candidate regarding teaching. The aim of this research was to analyze the interest in the teaching profession and the reasons that encourage the students of Master of Teaching in Secondary Education (specialty Physical Education) of the University of Granada, Sevilla and Jaen (Spain), to opt for the teaching profession, as well as determine their main ideas about the initial formation. For this purpose, a Questionnaire on interest in teaching and initial training (CIDFI) was applied (Pontes, Ariza \& Del Rey, 2010) in 217 students (154 men and 63 women, between 21 and 35 years old). The data revealed a predominant vocational tendency, especially in women, considering very important the knowledge of pedagogy, didactics and teaching programming, as well as the acquisition of skills to motivate the students to learn. In conclusion, as a proposal for improvement, it seems appropriate to include in the master activities that would make it possible to explain the previous ideas and motivations of future teachers, as well as these data should be taken into account in the design of the initial training, both general and specific, of future teachers of High school.
\end{abstract}

KEYWORDS: University Studies; Teacher Training Program; Physical Education; Secondary Education.

\section{Introducción}

La identidad profesional puede considerarse como un prisma a través del que analizar el modo en que los docentes sienten y ejercen su oficio (Bolivar, 2007). A nivel conceptual, la identidad docente puede abordarse a través de preguntas tales como ¿quién soy como profesor?, ¿cuáles son los significados vinculados a mí mismo en mi trabajo?, ¿qué tengo que hacer y por qué? (Contreras, Monereo, y Badía, 2010).

Como apuntan Muñoz y Ruiz de Miguel, (2017), el pensamiento de los docentes es un factor esencial en la investigación sobre la identidad profesional (Masuda, 2012), la cual es un constructo complejo y dinámico sobre el que influyen creencias, características personales, historias de aprendizaje, experiencias y actitudes previas, competencias 
profesionales, conocimientos y habilidades pedagógicas, entre otros (Pillen, Den Brok y Beijaard, 2013).

Este término, considerado dentro del ámbito de la enseñanza y la formación inicial del profesorado se relaciona con la percepción de la profesión, "determinando aspectos como la práctica docente, la forma en que se desarrolla la persona como profesor y sus actitudes hacia los cambios educativos" (Ariza, Sánchez, y Pontes, 2011: 242).

La identidad profesional docente es por tanto un aspecto esencial para el trabajo pedagógico, ya que guiará las acciones del docente, orientará sus valores y prácticas, dentro y fuera del aula y en relación a la comunidad educativa (Quilaqueo, Quintriqueo, y Riquelme, 2016).

Como señalan Ariza et al. (2011: 242) "La génesis de la identidad profesional docente viene determinada tanto por los motivos que explican el interés en el ejercicio de la profesión como por los cambios promovidos en las actitudes y creencias del candidato a profesor respecto a la enseñanza...". Todos los docentes de cualquier materia y nivel educativo, poseen actitudes, motivaciones e ideas previas sobre la docencia y la formación inicial (Campanario, 1998) que influyen en la formación de su identidad profesional docente (Marcelo, 2009) y que deben considerarse para la mejora del proceso de formación del profesorado (Pontes, Ariza, Serrano, y Sánchez, 2011).

Diversos estudios han señalado la existencia de numerosos motivos que incentivan el interés hacia la docencia (Córdoba, Ortega, y Pontes, 2009; Pontes et al., 2010; Pontes et al., 2011). Se han encontrado desde motivaciones altruistas relacionadas con el papel social de la educación y ligadas a la motivación intrínseca y la vocación docente, hasta motivos más pragmáticos centrados en los beneficios de la profesión docente, tales como la estabilidad y seguridad que ofrece el funcionariado, el salario o las vacaciones (Lissen, 2009; Pontes, Ariza, y del Rey, 2010). Otros motivos hacen referencia a la influencia de familiares o profesores en la dedicación y motivación docente (Ariza et al., 2011), o la motivación hacia el ejercicio docente de último recurso, guiados por las dificultades del mundo laboral y la escasez de salidas profesionales afines.

También resulta relevante y en relación con la identidad profesional la manera de entender y valorar la formación inicial del profesorado (Poyato, Pontes, y Oliva, 2017). En este caso se ha observado que la mayoría de los futuros docentes coinciden en la complejidad de la profesión y la necesidad de una adecuada formación inicial. No obstante, no todos valoran del mismo modo la importancia de la didáctica específica y los aspectos psicopedagógicos, la experiencia docente o el amplio dominio de los contenidos (Pontes et al., 2010).

Existen investigaciones que han relacionado de manera positiva la identidad profesional del docente con variables tan significativas como la eficacia en el ejercicio docente (Alsup, 2006), el compromiso como docente (Burn, 2007) o la capacidad para adaptarse y realizar cambios en el entorno escolar (Day, Stobart, Sammons, y Kington, 2007). Todo 
esto hace que la identidad profesional, la percepción que se tenga sobre la profesión docente y las motivaciones que los futuros docentes tienen hacia dicha labor, pueda ser considerado un objeto de estudio de carácter relevante (Ariza et al., 2011). No obstante, escasos estudios se han encontrado acerca de la identidad docente del profesorado especialista de Educación Física (EF) en Educación Secundaria Obligatoria (ESO).

Por todo ello, el objetivo del presente estudio es conocer el grado de interés por la profesión docente y la tipología de motivos que incitan al alumnado del Máster Universitario en Profesorado de Educación Secundaria Obligatoria y Bachillerato (especialidad Educación Física) formado en las Universidades de Sevilla, Granada y Jaén, a decantarse hacia la profesión docente, así como determinar las principales ideas previas acerca de la formación inicial necesaria para poder ejercer la docencia en dichos niveles educativos.

\section{Método}

La presente investigación se encuadra en el marco de un estudio no experimental y descriptivo de corte transversal.

\section{Participantes}

Se recogieron datos del alumnado del Máster Universitario en Profesorado de Educación Secundaria Obligatoria y Bachillerato en la especialidad de Educación Física, de las Universidades de Granada, Sevilla y Jaén, durante los cursos 2016-2017 y 2017-2018. La muestra estuvo compuesta por un total de 217 estudiantes: 154 hombres $(71 \%)$ y 63 mujeres (23\%), de entre 21 y 35 años, con una media de edad de 24,33 años (DT=2,59).

\section{Instrumento}

Se utilizó el Cuestionario sobre interés por la docencia y la formación inicial (CIDFI) (Ariza et al., 2011; Pontes et al., 2010). Dicho instrumento está compuesto por 19 items valorados en una escala Likert del 1 al 4 (nada de acuerdo, poco, bastante y muy de acuerdo), y distribuidos en dos apartados: Interés por la docencia (12 items) e Ideas previas sobre la formación docente (7 ítems). A su vez los ítems de Interés por la docencia, se agrupan en cinco dimensiones: Actitud vocacional, Ausencia de alternativas, Inducción por terceras personas, Condiciones laborales y Percepción del entorno. Mientras que en las Ideas previas sobre la formación docente se diferencian, según Pontes, Ariza y Del Rey (2010), ideas relacionadas con un Modelo transmisivo (se prioriza el conocimiento de los contenidos y la capacidad de transmitirlos), la 
Formación integral (relevancia del dominio psicoeducativo), la Formación empírica (práctica como eje central del proceso formativo, infravalorando formación psicoeducativa), y Cualidades Innatas (capacidad individual propia para comunicación y docencia). También se recogen datos sociodemográficos como la edad y el género.

\section{Procedimiento}

La recogida de datos se llevó a cabo durante el horario de clases. Los sujetos encuestados fueron informados acerca de los objetivos del estudio y aceptaron participar en el mismo previo consentimiento informado. Cumplimentaron el cuestionario tras recibir las instrucciones del encuestador, estando el investigador siempre presente para aclarar cualquier duda que pudiesen plantear los encuestados.

\section{Análisis estadístico}

Para el análisis de datos se ha utilizado el paquete estadístico SPSS versión 24.0. (SPSS, Inc., Chicago, IL). Se ha efectuado un análisis descriptivo obteniendo medias, desviación típica, frecuencias y porcentajes. Se usó el test de chi-cuadrado para analizar las posibles diferencias según el género del alumnado, fijando el nivel de significación en $\mathrm{p} \leq 0,05$.

\section{Resultados}

A continuación (tabla 1) se muestran los datos descriptivos de la muestra. Se observa que en todas las universidades es mayor el número de hombres que cursan esta titulación que el de mujeres. Igualmente, en todas las universidades, el porcentaje mayor de estudiantes que cursan el máster tiene una edad de entre 20 y 25 años.

Tabla 1.

Edad y género de los encuestados de cada universidad

\begin{tabular}{lccccccc}
\hline & \multicolumn{3}{c}{ Género $F(\%)$} & \multicolumn{5}{c}{ Rangos de edad F (\%) } \\
& Hombre & Mujer & Total & $\mathbf{2 0 - 2 5}$ & $\mathbf{2 6 - 3 0}$ & $+\mathbf{3 0}$ & Total \\
\hline Granada & 109 & 42 & 151 & 115 & 24 & $5(3,5)$ & 144 \\
& $(72,2)$ & $(27,8)$ & $(100)$ & $(79,9)$ & $(16,7)$ & & $(100)$ \\
Sevilla & $33(64,7)$ & 18 & 51 & $40(80)$ & $9(18)$ & $1(2)$ & 50 \\
& & $(35,3)$ & $(100)$ & & & & $(100)$ \\
Jaén & $12(80)$ & $3(20)$ & 15 & 8 & 6 & $0(0)$ & 14 \\
& & & $(100)$ & $(57,1)$ & $(42,9)$ & & \\
\hline
\end{tabular}


En la tabla 2 se detallan los resultados obtenidos en relación al interés por el ejercicio de la docencia, así como los valores de cada dimensión. Dichos datos dejan entrever el interés real del alumnado por la función docente $(3,65)$, siendo además el origen de ese interés de carácter más bien intrínseco, pues se han obtenido puntuaciones medias bajas en el interés debido a la ausencia de otras alternativas $(1,69)$ o las condiciones materiales y laborales beneficiosas del ejercicio de dicha profesión $(\mathrm{M}=2,02)$.

Cabe destacar también la influencia positiva que ejercieron algunos profesores $(3,17)$ a la hora de decantarse hacia la profesión docente. No obstante, aunque dicho interés se produce o inicia mayoritariamente al comienzo de los estudios universitarios $(2,72)$, también señalaron aspirar a otra profesión durante su carrera, aunque al finalizar los estudios la docencia les comenzó a parecer más interesante $(2,03)$.

En cuanto a la percepción del entorno docente, el alumnado la considera en cierta medida problemática $(3,33)$ y con una imagen que debería mejorarse $(2,95)$. Sin embargo, la consideran en mayor medida agradable y motivadora $(3,59)$.

Tabla 2.

Valores sobre Interés por la docencia del alumnado universitario

\begin{tabular}{lllll}
\hline Ittems del cuestionario & $M$ & $D T$ & Dimensiones & $M$ \\
\hline
\end{tabular}

A1 Tengo verdadero interés por ejercer la docencia en Educación Secundaria

$3,650,60$

A3 Mi interés por la docencia proviene desde que comencé los estudios 2,72 1,06 vocacional Actitud $\quad 3,32$ universitarios

A6 La profesión docente es agradable y motivadora por trabajar educando a 3,59 0,54 jóvenes

\begin{tabular}{lllll}
\hline $\begin{array}{l}\text { A2 Solo me interesa la docencia porque no } \\
\text { tengo otras salidas profesionales }\end{array}$ & 1,69 & 0,82 & $\begin{array}{l}\text { Ausencia de } \\
\text { alternativas }\end{array}$ & 1,69 \\
\hline
\end{tabular}

\begin{tabular}{llll}
\hline $\begin{array}{l}\text { A4 Durante estudios universitarios } \\
\text { aspiraba a otra profesión pero ahora me 2,03 }\end{array}$ & 0,99 & $\begin{array}{c}\text { Inducción por } \\
\text { terceras }\end{array}$ & 2,60 \\
interesa docencia & & personas \\
$\begin{array}{l}\text { A10 Algunos de mis profesores ejercieron } \\
\text { influencia positiva para interesarme en } 3,17\end{array}$ & 0,85 & \\
docencia & &
\end{tabular}

A5 La docencia solo me interesa por condiciones materiales: estabilidad, 2,02 0,81 Condiciones 2,13 vacaciones, etc. 
Motivos de interés por la docencia e identidad profesional del futuro profesorado de Educación Física. Análisis en los másteres universitarios de Sevilla, Granada y Jaén (España)

A12 Mi interés por la docencia tiene antecedentes familiares

laborales

$\begin{array}{lll}\text { A7 Los tiempos actuales no ofrecen buena } & \text { 2,95 } & 0,82 \\ \text { imagen de la docencia Secundaria } & \end{array}$

$2,251,19$

A8 La profesión docente es problemática

pero se pueden superar dificultades

$3,330,68$

A9 Cuando yo estudiaba Secundaria los

Percepción del 2,63

entorno

profesores tenían menos problemas que 2,46 0,84

ahora

A11 En mi círculo social no existe buena

opinión sobre profesión docente en 1,81 0,89

Secundaria

M: Media; DT: Desviación tipica

Atendiendo a las dimensiones expuestas en la tabla previa, se puede destacar por tanto la orientación del alumnado hacia una actitud vocacional por la docencia $(3,32)$, influenciada en muchos casos por docentes que les animaron a interesarse en la profesión $(2,60)$, a pesar de ser conscientes de las dificultades que presenta actualmente el entorno docente $(2,63)$.

Tabla 3.

Descripción del Interés por la docencia según el género del alumnado

\begin{tabular}{|c|c|c|c|c|c|c|c|c|c|}
\hline \multirow[b]{2}{*}{ Ítems del cuestionario } & \multicolumn{2}{|c|}{1} & \multicolumn{2}{|c|}{2} & \multicolumn{2}{|c|}{3} & \multicolumn{2}{|c|}{4} & \multirow[b]{2}{*}{$\mathbf{x}^{2}$} \\
\hline & $\mathbf{H}$ & $\mathbf{M}$ & $\mathbf{H}$ & $\mathbf{M}$ & $\mathbf{H}$ & $\mathbf{M}$ & $\mathbf{H}$ & $\mathbf{M}$ & \\
\hline $\begin{array}{l}\text { A1 Tengo verdadero interés por } \\
\text { ejercer la docencia en Educación } \\
\text { Secundaria }\end{array}$ & 0,0 & 1,6 & 3,9 & 7,9 & 22,7 & 27 & 73,4 & 63,5 & 0,184 \\
\hline $\begin{array}{l}\text { A3 Mi interés por la docencia proviene } \\
\text { desde que comencé los estudios } \\
\text { universitarios }\end{array}$ & 16,2 & 20,6 & 22,1 & 20,6 & 34,4 & 25,4 & 27,3 & 33,3 & 0,523 \\
\hline $\begin{array}{l}\text { A6 La profesión docente es agradable } \\
\text { y motivadora por trabajar educando a } \\
\text { jóvenes }\end{array}$ & 0,0 & 0,0 & 1,9 & 4,8 & 36,4 & 34,9 & 61,7 & 60,3 & 0,517 \\
\hline $\begin{array}{l}\text { A2 Solo me interesa la docencia } \\
\text { porque no tengo otras salidas } \\
\text { profesionales }\end{array}$ & 43,1 & 68,3 & 38,6 & 25,4 & $12 \% 4$ & 6,3 & 5,9 & 0,0 & $0,005^{*}$ \\
\hline $\begin{array}{l}\text { A4 Durante estudios universitarios } \\
\text { aspiraba a otra profesión pero ahora } \\
\text { me interesa docencia }\end{array}$ & 33,3 & 50,8 & 28,8 & 28,6 & 26,1 & 19,0 & 11,8 & 1,6 & 0,021 * \\
\hline
\end{tabular}


A10 Algunos de mis profesores ejercieron influencia positiva para $5,8 \quad 0,0 \quad 18,2 \quad 14,3 \quad 33,1 \quad 46,0 \quad 42,9 \quad 39,7 \quad 0,102$ interesarme en docencia

A5 La docencia solo me interesa por

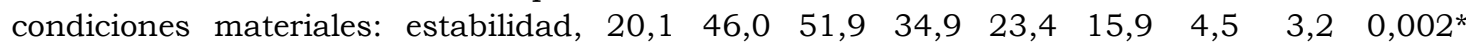
vacaciones

A12 Mi interés por la docencia tiene antecedentes familiares

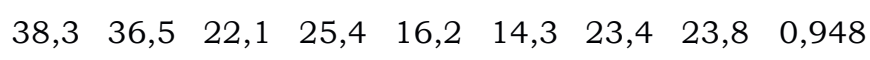

A7 Los tiempos actuales no ofrecen buena imagen de la docencia $\quad 5,2 \quad 3,2 \quad 23,4 \quad 21,0 \quad 47,4 \quad 41,9 \quad 24,0 \quad 33,9 \quad 0,500$ Secundaria

A8 La profesión docente es problemática pero se pueden superar $\quad 0,0 \quad 0,0 \quad 10,5 \quad 16,1 \quad 43,1 \quad 41,9 \quad 46,4 \quad 41,9 \quad 0,501$ dificultades

A9 Cuando yo estudiaba Secundaria los profesores tenían menos $10,5 \quad 12,9 \quad 44,4 \quad 40,3 \quad 33,3 \quad 35,5 \quad 11,8 \quad 11,3 \quad 0,924$ problemas que ahora

A11 En mi círculo social no existe buena opinión sobre profesión $44,7 \quad 46,0 \quad 35,5 \quad 30,2 \quad 13,8 \quad 19,0 \quad 5,9 \quad 4,8 \quad 0,730$ docente en Secundaria

H: Hombre; M: Mujer; 1(Nada de acuerdo), 2(Poco de acuerdo), 3(Bastante de acuerdo), 4(Muy de acuerdo); * $p \leq 0,05$

Si analizamos el Interés por la docencia en función del género del alumnado (tabla 3), se observa que solo existen diferencias significativas en los ítems A2, A4 y A5. Según estos datos, las mujeres se muestran más en desacuerdo que los hombres respecto a la afirmación "Solo me interesa la docencia porque no tengo otras salidas profesionales", por lo que supone una salida profesional prioritaria especialmente para ellas. En consonancia con lo anterior, las chicas también se muestran más en desacuerdo con la afirmación "Durante los estudios universitarios aspiraba a otra profesión pero ahora me interesa docencia". Siguiendo esta misma línea, ellas están también más disconformes que ellos con el interés por la docencia debido a las condiciones materiales (estabilidad, vacaciones, etc.).

Las ideas previas acerca de la formación docente que tienen los futuros profesores de EF de Secundaria se muestran en la siguiente tabla (4). 
Motivos de interés por la docencia e identidad profesional del futuro profesorado de Educación Física. Análisis en los másteres universitarios de Sevilla, Granada y Jaén (España)

Tabla 4.

Valores sobre las Ideas previas sobre la formación docente del alumnado universitario

\begin{tabular}{lllll} 
Ítems del cuestionario & $M$ & $D T$ & Dimensiones & $M$ \\
\hline
\end{tabular}

B1 Para ser profesor solo importa tener un

amplio conocimiento de los contenidos y $1,75 \quad 0,71$

enseñarlos con claridad

B6 El profesorado de Secundaria, además de

Modelo

conocer bien la materia, debe aprender a

programar su actividad y adquirir destrezas,

transmisivo

2,69

técnicas e instrumentos de comunicación

B2 Antes de ser profesor es necesario adquirir

conocimientos profundos de psicología, pedagogía y didáctica específica de la propia 3,32 0,65

materia que se ha de enseñar

Formación

integral

B4 Para motivar a los alumnos es necesario

adquirir ciertas destrezas de la profesión 3,47 0,55

docente

B3 La formación docente del profesor no se

adquiere mediante cursos de pedagogía o 2,61 0,84

didáctica

Formación

empirica

2,76

B7 Solo se aprende a ser buen profesor a través de la experiencia

$2,910,80$

B5 Hay personas con cualidades innatas para

la comunicación y docencia, tales destrezas no 3,00 0,79 $\begin{gathered}\text { Cualidades } \\ \text { innatas }\end{gathered} \quad 3,00$ se adquieren en cursos

M: Media; DT: Desviación tipica

Los datos de la tabla anterior (4) nos muestran que las ideas más extendidas entre el futuro profesorado son las relacionadas con la Formación integral como docente $(3,39)$, ya que según la población encuestada, para ser profesor de Secundaria es necesario adquirir importantes conocimientos de pedagogía y didáctica, $(3,32)$, así como adquirir destrezas docentes que permitan motivar a los alumnos por el aprendizaje $(3,47)$. No obstante, resulta especialmente relevante la valoración que el alumnado realiza de las posibles cualidades innatas para la docencia $(3,00)$.

Es también llamativo la orientación similar que muestran hacia la Formación empírica $(2,76)$ y el Modelo transmisivo $(2,69)$. La importancia que el alumnado concede a la práctica docente resulta limitada a tenor de las puntuaciones de los items B3 y B7 (2,61y 2,91 respectivamente). Dentro del Modelo transmisivo valoran en mayor medida la capacidad 
comunicativa $(3,63)$, sobre el bagaje de conocimientos propios de la materia $(1,75)$.

Tabla 5.

Análisis de las Ideas previas sobre la formación docente según el género del alumnado

\begin{tabular}{|c|c|c|c|c|c|c|c|c|c|}
\hline \multirow[b]{2}{*}{ Ítems del cuestionario } & \multicolumn{2}{|c|}{1} & \multicolumn{2}{|c|}{2} & \multicolumn{2}{|c|}{3} & \multicolumn{2}{|c|}{4} & \\
\hline & $\mathbf{H}$ & $\mathbf{M}$ & $\mathbf{H}$ & $\mathbf{M}$ & $\mathbf{H}$ & $\mathbf{M}$ & $\mathbf{H}$ & $\mathbf{M}$ & $\mathbf{x}^{2}$ \\
\hline
\end{tabular}

B1 Para ser profesor solo importa tener

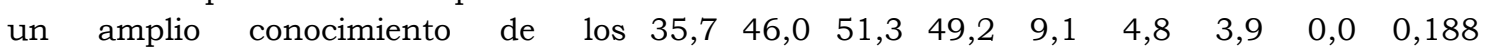
contenidos y enseñarlos con claridad

B6 El profesorado de Secundaria, además de conocer bien la materia, debe $\begin{array}{llllllllll}\text { aprender a programar su actividad y } & 0,7 & 0,0 & 1,3 & 3,2 & 29,4 & 39,7 & 68,6 & 57,1 & 0,307\end{array}$ adquirir destrezas, técnicas e instrumentos de comunicación

B2 Antes de ser profesor es necesario adquirir conocimientos profundos de

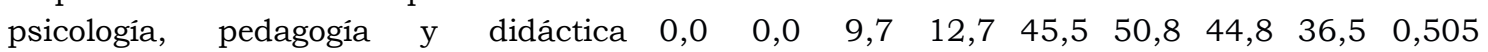
específica de la propia materia que se ha de enseñar

B4 Para motivar a los alumnos es

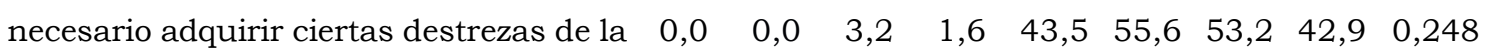
profesión docente

B3 La formación docente del profesor no se adquiere mediante cursos de $5,2 \quad 11,1 \quad 37,3 \quad 52,4 \quad 39,9 \quad 23,8 \quad 17,6 \quad 12,7 \quad 0,035^{*}$ pedagogía o didáctica

B7 Solo se aprende a ser buen profesor a través de la experiencia

$2,6 \quad 4,8 \quad 26,6 \quad 30,2 \quad 44,2 \quad 42,9 \quad 26,6 \quad 22,2 \quad 0,751$

B5 Hay personas con cualidades innatas para la comunicación y docencia, tales $\quad 2,0 \quad 3,2 \quad 19,0 \quad 38,1 \quad 44,4 \quad 42,9 \quad 34,6 \quad 15,9 \quad 0,006^{*}$ destrezas no se adquieren en cursos

H: Hombre; M: Mujer; 1(Nada de acuerdo), 2(Poco de acuerdo), 3(Bastante de acuerdo), 4(Muy de acuerdo); * $p \leq 0,05$

Si analizamos las Ideas previas sobre la formación docente en función del género del alumnado (tabla 5), se observa que solo existen diferencias significativas en los ítems B3 y B5. Según estos datos, los hombres conceden menor importancia a la formación a través de los cursos de pedagogía y didáctica $(57,5 \%$ de los hombres están bastante y muy de acuerdo con la afirmación, frente al $36,5 \%$ de las mujeres), y mayor importancia que las mujeres a las posibles cualidades innatas que los

308 ISSN 0213-8646 | E-ISSN 2530-3791 • Revista Interuniversitaria de Formación del Profesorado, 95 (34.2) (2020), 299-314 
Motivos de interés por la docencia e identidad profesional del futuro profesorado de Educación Física. Análisis en los másteres universitarios de Sevilla, Granada y Jaén (España)

sujetos pueden presentar para la función docente $(79 \%$ de los hombres están bastante y muy de acuerdo con la afirmación, frente al 58,8\% de las mujeres).

\section{Discusión y conclusión}

Antes de comenzar la discusión acerca del interés por la docencia o las ideas previas, cabe destacar un par de observaciones en relación a las características demográficas del alumnado que cursa el máster. Respecto al género, se observa que en todas las universidades es mayor el número de hombres que de mujeres que cursan esta titulación, lo que puede dejar entrever esa orientación masculina hacia la Educación Física que aún puede seguir imperando en los estudios universitarios. En cuanto a la edad, predominan estudiantes de entre 20 y 25 años. Esto supone que estudian esta titulación nada más acabar la carrera, y puede significar que la docencia constituye una de sus primeras opciones laborales, sin siquiera explorar o tantear otras previas.

Los datos del cuestionario respecto a las Ideas previas del alumnado han mostrado un interés real de los futuros profesores hacia el ejercicio de la docencia, siendo además el origen de ese interés de carácter más intrínseco y vocacional que extrínseco o pragmático.

Estos datos no coinciden con los obtenidos por Pontes et al., (2010) en la Universidad de Córdoba; ni con los de Córdoba et al. (2009) o Poyato et al. (2017) de los estudiantes de carreras de ciencias, donde la docencia en Educación Secundaria no constituía un elemento preferente, sino que cursaban el Máster de Educación Secundaria como una opción más de futuro. No obstante, bien es cierto que tradicionalmente y desde un punto de vista más social, los estudios relacionados con la Educación Física se han asociado en mayor medida al ámbito educativo que otras disciplinas quizás más científicas, como las referidas anteriormente por Pontes et al., (2010) y Poyato et al. (2017). Así, tradicionalmente en las antiguas carreras de Magisterio existía la especialidad en Educación Física, al igual que existe hoy en día la mención de Educación Física en el Grado de Educación Primaria. No podemos olvidar que el alumnado graduado en Educación Primaria tiene acceso actualmente al Máster Universitario en Profesorado de Educación Secundaria Obligatoria y Bachillerato. Igualmente, en la formación del alumnado del Grado en Ciencias de la Actividad Física y el Deporte existen asignaturas específicas de formación en materia docente, como puede ser la de Didáctica o Aprendizaje y Enseñanza, entre otras.

La investigación de Latorre y Blanco (2014), con los estudiantes del Máster de Educación Secundaria de la Universidad de Granada aporta datos similares en cuanto a la orientación docente. En estos casos el alumnado, a pesar de ser consciente de que en los tiempos actuales no se ofrece una buena imagen de la docencia, se encuentra bastante interesado por esta salida profesional. Aunque ellos mostraban interés 
principalmente por razones más extrinsecas, como la ausencia de otras salidas profesionales o las condiciones materiales de la profesión. Igualmente, como ocurre en la presente investigación, las posturas de los estudiantes a la hora de elegir la docencia como salida profesional desde un primer momento o durante la realización de la carrera universitaria se encontraban divididas, sintiendo los futuros docentes una vocación temprana o tardía según el caso. Datos parecidos obtuvieron Pontes et al. (2011) y Pontes et al. (2010), aunque en estos casos la mayor parte del alumnado reconoció que su interés hacia la docencia se produjo al finalizar la carrera universitaria.

Respecto a la imagen de la profesión docente, los futuros profesores de Educación Física de Secundaria la han considerado en cierta medida como problemática, aunque la consideran en mayor medida aún como agradable y motivadora. Esta opinión coincide con la recogida por Pontes et al. (2010), pero contrasta con los datos recogidos en otras investigaciones previas (Pontes y Serrano, 2008; Lissen, 2009), donde el alumnado se mostraba más pesimista todavia respecto a la opinión de la profesión docente. En este sentido, la postura más optimista y motivada del futuro profesorado de Educación Física, puede volver a estar relacionada con esa mayor relación social que tradicionalmente ha tenido y parece ser que sigue teniendo la Educación Física con la formación y educación en general.

Se ha observado también la influencia positiva ejercida por algunos profesores en el interés hacia la profesión docente, hecho ya recogido en otros estudios (Latorre y Blanco 2014; Pontes et al., 2010).

Al analizar los datos del Interés por la docencia según el género del alumnado, se recoge la idea de que la vocación docente en las mujeres es algo mayor en este caso que en los hombres, pues contemplan la docencia como opción prioritaria y como salida laboral desde el comienzo de la carrera. De hecho, se muestran incluso menos interesadas que ellos por las condiciones materiales de dicha profesión. Estos datos sin embargo no coinciden con los de Ariza, Sánchez y Pontes (2011), quienes no obtuvieron ninguna diferencia significativa en función del género respecto a la docencia como salida profesional de los estudiantes de Humanidades y Ciencias Sociales en Córdoba. No obstante, estos autores señalaban que para los hombres la percepción del entorno tenía mayor poder decisorio que para las mujeres. Mientras que para ellas las condiciones laborales condicionaban la elección de la salida profesional en mayor medida, justo la tendencia inversa observada en el presente estudio. No obstante, estas tendencias pueden estar influidas por otros muchos factores como la edad o la especialidad estudiada, no habiendo encontrado entre la bibliografia revisada otros datos comparables con el alumnado especialista en EF. En esta línea, quizás fuera muy interesante estudiar a través de técnicas cualitativas que permitieran profundizar, bien a través de entrevistas u otras, en las reflexiones y motivaciones más profundas de hombres y mujeres. 
En cuanto a las ideas previas acerca de la formación inicial del profesorado, cabe destacar que los resultados obtenidos difieren enormemente a los de Pontes et al. (2010) o Poyato et al. (2017). En estos casos los estudiantes apenas valoraban la importancia de una formación inicial docente integrada por conocimientos didácticos y prácticos e infravaloraban la formación psicopedagógica inicial, considerando por el contrario más importante la transmisión de conocimientos disciplinares (Pontes et al., 2010). En el presente estudio, los datos obtenidos concuerdan enormemente con los registrados por Latorre y Blanco (2014), con los estudiantes del Máster de Educación Secundaria de la Universidad de Granada. En estos casos el alumnado ha considerado muy importantes los conocimientos de psicología, pedagogía y didáctica, así como la adquisición de destrezas para motivar a los alumnos por el aprendizaje. No basta con saber muchos contenidos y dominar absolutamente la materia, es necesario también saber programar y adquirir destrezas propias de la profesión docente.

Igualmente, en consonancia con el estudio previamente citado, llama la atención la idea generalizada del alumnado de que hay personas con cualidades innatas para el ejercicio de la docencia, que resalta por el carácter determinista y genetista acerca del posible perfil ideal del docente. Además, según los datos del presente estudio, son los hombres quienes conceden menos importancia a la formación a través de los cursos de pedagogía y didáctica, pero más importancia que las mujeres a dichas cualidades innatas para la función docente. Junto con la anterior línea citada para revisar las diferencias de género, referente a la motivación hacia la educación como salida profesional, este podría ser otro punto muy importante para incluir. Seria interesante profundizar a través de herramientas cualitativas en el análisis del sesgo de género en la profesión docente y el perfil del/la "profesor/a ideal", así como de la importancia que conceden a la formación del futuro profesorado o a las características innatas que puedan considerar debiera tener ese "modelo ideal".

Para finalizar, considerando todo el análisis de los resultados obtenidos, se puede afirmar que el alumnado que cursa el Máster de Profesorado de Educación Secundaria (especialidad EF) se encuentra matriculado en dicho curso por motivos más intrínsecos que pragmáticos y considera necesaria la adquisición de una sólida formación inicial docente para el correcto ejercicio de la profesión y la contribución a la calidad de la Educación Secundaria Obligatoria.

Como propuesta de mejora parece conveniente incluir dentro del máster actividades que permitiesen explicitar las ideas previas y motivaciones de los futuros docentes, así como estos datos deberian tenerse en cuenta en el diseño de la formación inicial, tanto general como específica, de los futuros docentes de Secundaria.

En cualquier caso, es necesario reseñar que los resultados del presente estudio deben tomarse con cautela por las limitaciones del mismo. Por un lado, la muestra no ha sido demasiado numerosa, por lo 
que serán necesarios estudios posteriores para ampliar y generalizar conclusiones. Igualmente, ha sido compleja la comparación de los datos debido a que no existe demasiada bibliografia en esta linea de investigación que aporte datos similares y comparables. Sobre todo teniendo en cuenta las especialidades concretas del Máster de Secundaria, para evitar posibles sesgos según los perfiles del alumnado. Sería interesante continuar avanzando en esta línea para poder diferenciar resultados en función de cada especialidad. Ha de tenerse en cuenta que ya algunos de los estudiantes de este máster con la especialidad de Educación Física pueden provenir del Grado en Educación Primaria (Dopico, 2016), por lo que dicha orientación hacia la función docente estaría mucho más marcada que en otras especialidades. Sería igualmente interesante analizar estas motivaciones e ideas previas de los futuros docentes de Educación Física teniendo en cuenta las posibles diferencias entre los profesores de enseñanza Primaria y Secundaria.

\section{Referencias}

Alsup, J. (2006). Teacher identity discourses: Negotiating personal and professional spaces. Mahwah, N.J.: Lawrence Erlbaum Associates. Recuperado de https://books.google.es/books?hl=es\&lr=\&id=d2RAgAAQBAJ\&oi=fnd\&pg=PP1\&dq=Alsup,+J.+(2006).+Teacher+ident ity+discourses:+Negotiating+personal+and+professional + spaces. $+\mathrm{M}$ ahwah,+N.J.:+Lawrence+Erlbaum+Associates.\&ots=1DLQDm44a1\& sig=eIYdlD7eSPdFBzpaIVLSL4_F-Hw\#v=onepage\&q\&f=false

Ariza, L., Sánchez, F.L. y Pontes, A. (2011). Motivos de interés por la docencia e identidad profesional. Una aproximación a partir de la opinión del futuro profesorado de Humanidades y Ciencias Sociales. Profesorado, 15(1), 1-22. Recuperado de https:/ / recyt.fecyt.es/index.php/profesorado/article/view/43509

Asensio Muñoz, I., y Ruiz de Miguel, C. (2017). Medida y evaluación de las creencias sobre la profesión de los maestros en formación. Revista Electrónica Interuniversitaria De Formación Del Profesorado, 20(3), 79-

91.https: / / doi.org/ 10.6018/reifop.20.3.265231

Bolivar, A. (2007). The Initial Training of Secondary School Teachers and their Professional Identity. ESE: Estudios sobre educación, 12, 13-30. Recuperado de https:/ / dadun.unav.edu/bitstream/10171/8988/1/12\%20Estudio s\%20Ea.pdf

Burn, K. (2007). Professional knowledge and identity in a contested 
Motivos de interés por la docencia e identidad profesional del futuro profesorado de Educación Física. Análisis en los másteres universitarios de Sevilla, Granada y Jaén (España)

discipline: challenges for student teachers and teacher educators. Oxford Review of Education, 33(4), 445-467. Recuperado de https://www.tandfonline.com/doi/abs/10.1080/03054980701450 886

Campanario, J.M. (1998). ¿Quiénes son, qué piensan y qué saben los futuros maestros y profesores de ciencias?: una revisión de estudios recientes. Revista Interuniversitaria de Formación del Profesorado, 33,121-140. Recuperado de https://dialnet.unirioja.es/servlet/articulo?codigo=117999

Contreras, C., Monereo, C. y Badia, A. (2010). Explorando en la identidad: ¿Cómo enfrentan los docentes universitarios los incidentes criticos que ocurren en las aulas de formacion de futuros profesores? Estudios Pedagógicos, XXXVI(2), 63-81. Recuperado de https://scielo.conicyt.cl/pdf/estped/v36n2/art04.pdf

Córdoba, F., Ortega, R. y Pontes, A. (2009). Universitarios de ciencias ante la docencia en Educación Secundaria como expectativa profesional. Revista de Educación, 348, 401-421. Recuperado de http://www.revistaeducacion.mec.es/re348/re348_17.pdf

Day, C., Stobart, G., Sammons, P. y Kington, A. (2007). Variations in the work and lives of teachers: relative and relational effectiveness. Teachers and Teaching: Theory and Practice, 12(2), 169-192. Recuperado https://www.tandfonline.com/doi/abs/10.1080/13450600500467 381

Dopico, M. (2016) Estudio descriptivo sobre la formación inicial de los/as maestros y maestras de Educación Física escolar. Sportis Sci J, 2 (2), 188-205. Recuperado http://dx.doi.org/10.17979/sportis.2016.2.2.1429

Latorre, M.J. y Blanco, F.J. (2014). Percepciones del profesorado en formación sobre la formación docente. En Del Valle, M.E. (Ed.), Experiencias en docencia superior (pp. 343-362). Madrid, España: ACCI.

Lissen, E. (2009). Mitos y realidades en la carrera docente. Revista de Educación, 348, 465-488. Recuperado de https://idus.us.es/xmlui/handle/11441/47068

Marcelo, C. (2009). Formalidad e Informalidad en el oficio de aprender a enseñar. Revista de Educación, 350, 31-55. Recuperado de https://idus.us.es/xmlui/bitstream/handle/11441/31362/Formal idad_e_informalidad.pdf?sequence $=1$ 
Masuda, A. (2012). Critical literacy and teacher identities: A discursive site of struggle. Critical Inquiry in Language Studies, 9(3), 220-246.

Pillen, M.T., Den Brok, P.J. y Beijaard, D. (2013). Profiles and change in beginning teachers' professional identity Tensions. Teaching and Teacher Education, 34, 86-97.

Pontes, A., Ariza, L. y Del Rey, R. (2010). Identidad profesional docente en aspirantes a profesorado de enseñanza secundaria. Psychology, Society, \& Education, 2(2), 131-142. Recuperado de http://repositorio.ual.es/bitstream/handle/10835/2877/131142. pdf? sequence $=1 \&$ is Allowed $=\mathrm{y}$

Pontes, A., Ariza, L., Serrano, R. y Sánchez, FJ. (2011). Interés por la docencia entre aspirantes a profesores de Ciencia y Tecnología al comenzar el proceso de formación inicial. Revista Eureka sobre Enseñanza y Divulgación de las Ciencias 8 (2), 180-195. Recuperado de

http:/ /rodin.uca.es/xmlui/bitstream/handle/10498/10852/4_Pon tes_et_al_2011.pdf?sequence $=6$

Pontes, A., y Serrano, R. (2008). Actitudes e ideas previas sobre la docencia y la formación docente en los aspirantes a profesores de ciencias experimentales. En R. Jiménez Liso (Eds.), Ciencias para el mundo contemporáneo y formación del profesorado en didáctica de las ciencias experimentales (pp.458-467). Almería: Editorial Universidad de Almería.

Poyato, FJ., Pontes, A. y Oliva, JM (2017). Los fines de la educación científica y la formación inicial del profesorado de secundaria. REEC: Revista electrónica de enseñanza de las ciencias, 16(1), 28-46. Recuperado de http://reec.uvigo.es/volumenes/volumen16/REEC_16_1_2_ex1007 .pdf

Quilaqueo, D., Quintriqueo, S. y Riquelme, E. (2016). Identidad Profesional Docente: Práctica Pedagógica en Contexto Mapuche. Estudios Pedagógicos, XLII(2), 269-284. Recuperado de https://scielo.conicyt.cl/pdf/estped/v42n2/art15.pdf 